\title{
7 The Bloke - Fashion made in Düsseldorf
}

Schon Giorgio Armani stellte treffend fest: „Wenn ein Anzug auffällt, ist man schlecht angezogen." Das ändert ein junges Unternehmen in Düsseldorf namens The Bloke, ein Herrenausstatter der nach Maß anfertigt. Anpassungen erfolgen individuell auf den Kunden zugeschnitten. Man nimmt sich Zeit für Beratung, Auswahl und Perfektion. Doch auch nach einer erfolgreichen Gründung und Etablierung am Markt gibt es viel zu tun. Dann entsteht unweigerlich die Frage, ob eine Expansionsstrategie und wenn ja, welche sich für das Unternehmen lohnt. Soll es neue Wege explorativ ergründen oder soll es bestehende Strukturen exploitativ ausreizen? Welche Wege führen zu einem erfolgreichen Wachstum?

Der Name „The Bloke“ bedeutet übersetzt so viel wie „Der Kerl“. Der Stil des seit 2013 bestehenden kreativen Modelabels hebt sich von anderen Anbietern über seine Qualität und Individualität ab. Sein Fokus ist auf ein Must-have im Kleiderschrank eines Mannes gerichtet: den Anzug. Ganz gleich, ob im Beruf, zu gesellschaftlichen Anlässen oder auf der eigenen Hochzeit - einen gutsitzenden, schicken Anzug benötigt jeder Mann. Der Anzug ist, ob alternativ oder ganz allgemein, ein Symbol, das in westlichen Kulturen für Macht, Reichtum und Eleganz steht. Er kann viele verschiedene Formen haben und zeichnet sich durch eine Liebe zum Detail aus: von der Größe des Revers über die Anzahl der Taschen bis hin zum Material für die Herstellung der Knöpfe gilt eine besondere Aufmerksamkeit.

The Bloke versucht, sich insbesondere durch eine hohe Materialqualität sowie über einen umfassenden Service von anderen Wettbewerbern abzuheben. Das Ziel des Unternehmens ist es, für jeden Kunden das perfekte Outfit zu designen. Es gibt keine fertige Kollektion, die jemandem übergestülpt wird, jeder erhält genau das, was zu ihm passt. „Es gibt nicht den typischen The Bloke-Geschmack, sondern in der Beratung versuchen wir herauszufinden, was der Kunde will, welche Wünsche und Bedürfnisse er hat. Uns geht es darum, den persönlichen Geschmack des Kunden zu verstehen“, sagt Gründerin Anna Ullrich-Cattien. „Für uns ist es wichtig, dass der Kunde hier reinkommt und ein gesamtes Einkaufserlebnis hat. Er bekommt hier seinen Kaffee, sein Feierabendbier und er soll sich wohlfühlen. Wir schaffen eine Atmosphäre, in der wir uns voll und ganz auf den Kunden und seine Wünsche konzentrieren können.“

Dabei ist der finanzielle Sprung von einem Anzug „,on der Stange“ zu einem von The Bloke nicht so enorm groß, wie so mancher denken könnte. Ein maßgeschneidertes Modell kostet ab 700 Euro, der Kunde verlässt The Bloke mit einem durchschnittlichen Einkaufswert in Höhe von rund 1.000 Euro. Es werden keine 
traditionellen Vollmaßanzüge angeboten, deren Preise auch 4.000 Euro betragen können, sondern ausschließlich Maßkonfektion. Als Basis dienen Grundschnitte, sogenannte Schlupfgrößen, die individuell angepasst werden. Für einen Vollmaßanzug wird der Kunde von Grund auf vermessen, dann fertigt ein Techniker eine Zeichnung an, anhand derer der Schnitt entworfen wird. The Bloke stützt sich hingegen auf einen bereits bestehenden Entwurf, der einschließlich Details an den jeweiligen Kunden angepasst wird. Die Anzüge schneidert das Unternehmen nicht selbst, sondern eine sorgfältig ausgewählte Näherei in Marokko. Die Palette der zur Auswahl angebotenen Anzugstoffe stellt The Bloke selbst zusammen und achtet dabei auf aktuelle Modetrends und eine ausgezeichnete Stoffqualität. Der Kunde hat die zwischen Stoffen aus Großbritannien oder Italien. Ein sehr hohes Servicelevel sowie individuelle Leistungen sind es also, die The Bloke von anderen Maßschneidern abheben. Es wird je nach Figur, Typ und Geschmack des Mannes beraten und angepasst - und das zu (fast) jeder Zeit!

\section{Vom Herrenausstatter zum Maßkonfektionär}

Herrenausstatter haben sich im 19. und 20. Jahrhundert als eine Spezialisierung von Schneidereien und Bekleidungsgeschäften etabliert. Während klassische Herrenausstatter gegenwärtig laut Branchenexperten ${ }^{1}$ vom Niedergang bedroht sind, werden Maßschneidereien weiterhin nachgefragt. Eigenmarken, Service und Beratung sind hier die klassischen Erfolgsfaktoren, die dem stationären Handel immer wieder anempfohlen werden, um sich im Wettbewerb zu behaupten.

Die Maßkonfektion als eigenständiger Zweig ist jedoch ein relativ kleiner Markt. Branchenexperten schätzten den Anteil am deutschen Handelsumsatz mit Herrenbekleidung auf ca. 5 Prozent vom Gesamtvolumen von zuletzt 7,1 Milliarden Euro. ${ }^{2}$ In Deutschland gibt es knapp 1.800 Maßschneider. ${ }^{3}$ Der klassische Maßschneider nimmt bis zu 30 Maße pro Person, seine Arbeitszeit für einen Anzug beträgt bis zu 80 Stunden - der Anzug kann dann bis zu 3.500 Euro kosten. Die Maßkonfektion nutzt Schlupfgrößen wie „regular size“ oder „slim fit“ als Vorlage und verlagert die Näharbeiten ins Ausland. So geht auch The Bloke vor. Seit mehr als zehn Jahren ist diese Art von Maßkonfektion ein Trend: Fertige Schnitte

1 Vgl. Oess, M. (2017). Da maßte nix! So arbeitet die Branche. FashionToday, Ausgabe März 2017. 2 Vgl. Weishaupt, G. (2015). Messen, schneidern, kaufen. Handelsblatt, 30.09.2015 (188), S. 24. 3 Vgl. Weishaupt, G. (2015). Messen, schneidern, kaufen. Handelsblatt, 30.09.2015 (188), S. 24. 
werden kundenindividuell angepasst und trotzdem zu Preisen ähnlich wie bei Anzügen „,von der Stange“ gefertigt.

Während sich in Nachbarländern wie Belgien und die Niederlande die Maßkonfektionäre in den Straßen aneinanderreihen, war zur Zeit der Gründung von The Bloke diese Art der Maßkonfektion in Deutschland nur vereinzelt in den großen Städten anzutreffen. Doch die Nische wächst nun auch hier. Neben dem seit fast 20 Jahren in Düsseldorf ansässigen Maßkonfektionär Cove expandierten Anbieter aus dem Süden Deutschlands wie DOLZER und KUHN nach NordrheinWestfalen. Europas Marktführer, die belgische SCABAL-Gruppe, meldet für ihr deutsches Tochterunternehmen Tailor Hoff in Saarbrücken ein Umsatzplus von 12 Prozent. DOLZER wächst ebenso wie The Bloke beständig im zweistelligen Prozentbereich.

Während der Markt für Damenmode sehr schnelllebig und dynamisch ist und sich Kollektionen in der Regel mehrfach im Jahr ändern, ist der Markt für Herrenkonfektionäre im Vergleich relativ stabil. Der durchschnittliche Businesskunde kauft drei bis vier Anzüge pro Jahr. Diese werden in der Regel nicht „aufgetragen“, sondern ergänzend zu bestehenden Stücken gekauft. Entsprechend wenig dynamisch sind auch die Modetrends in diesem Bereich. „Umso wichtiger ist es, dass man Trends im Blick behält, die Schnitte modernisiert und sich anpasst an dem, was jetzt modern ist. Der trendbewusste Kunde erwartet natürlich insbesondere von einem Maßkonfektionär, dass er die neuen Details und Schnitte umsetzen kann.“, so die Gründerin von The Bloke.

Mittlerweile wird der Trend in Deutschland und insbesondere in der Modestadt Düsseldorf immer beliebter. Traditionsunternehmen wie Cove, die seit 1990 in der Maßkonfektion tätig sind und ihren Hauptsitz nach Düsseldorf verlegt haben, sind nicht nur mittlerweile deutschlandweit in Filialen vertreten, sondern entwickeln zudem neue Label. Sons of Savile Row beispielsweise möchte insbesondere eine jüngere Kundschaft gewinnen. Sogar Kaufhausketten folgen dem Trend. Während HUGO BOSS schon lange Zeit Maßanzüge anbietet, fertigt seit 2018 auch Peek \& Cloppenburg Anzüge nach Maß an und stellt Kunden dafür einen Stilberater zur Seite.

Aber auch unkonventionelle neue Wettbewerber spielen mit. Beispielsweise kann man über die Plattform von tailorjack Maßanzüge automatisiert bestellen. Der Kunde designt hier seinen eigenen Anzug, indem er seine Maße online eingibt. Die Fertigung erfolgt in Thailand von Hand. Unweit von The Bloke entfernt hat im Stadtzentrum Düsseldorfs das aus Paris stammende ATELIER NA sein zweites Geschäft in Deutschland eröffnet. Ein selbst entwickelter und patentierter Bodyscanner erspart das traditionelle Vermessen gänzlich. Für den Fall, dass Laser und Computer sich doch mal vertun sollten, sind in jedem Geschäft noch traditionelle Schneider anzutreffen, welche die Fehler kostenlos beheben. Kun- 
denmaße und -profile sind in der Datenbank gespeichert und können für Folgeeinkäufe abgerufen werden, um erneute Vermessungen zu vermeiden. Passend zum Trend der Maßkonfektion trat im August 2018 das japanische Start-up ZOZO in den deutschen Markt ein. Hier erfolgt die Vermessung mittels komplexer 3-DTechnologie per Smartphone. Auf dieser Basis werden dann Maßanzüge und andere Kleidungsstücke erstellt.

\section{Selbst ist die Frau}

Ullrich-Cattien stammt nicht in klassischer Weise aus einer Schneiderei oder dem Design, sondern hat Betriebswirtschaft mit Schwerpunkt Marketing studiert. Sie sammelte einige Jahre Erfahrungen in der Industrie. Dort war sie als Produktmanagerin tätig und hat ihre Marketingkompetenzen ausbauen können, welche ihr beim Aufbau der Marke The Bloke halfen. Gereift war die Idee aber schon lange vorher, nicht zuletzt inspiriert von ihrem Mann, der bei einem großen Modehersteller tätig ist. Bis zur Gründung von The Bloke musste die Gründerin mit ihrem Mann in die Niederlande oder Belgien fahren, um Anzüge für ihn bei einem Maßkonfektionär schneidern zu lassen. Da sich Ullrich-Cattien schon längere Zeit überlegte, sich selbstständig zu machen, fasste sie den Entschluss, die Marktlücke der Maßkonfektion in Düsseldorf zu schließen und The Bloke zu gründen.

Anfang 2013 arbeitete die Gründerin den Businessplan aus; finanziert wurde die Idee neben dem eingebrachten Eigenkapital lediglich mit einem Sparkassen-Gründerkredit. Ihr erstes Ladengeschäft eröffnete sie im Dezember 2013 im Stadtteil Pempelfort in einer ruhigen Seitenstraße. Laufkundschaft gibt es hier kaum, viel Fluktuation ist jedoch im Gegensatz zu großen Modeketten auch nicht gewünscht. Service ist ein Grundgedanke bei The Bloke, und dazu gehört die umfassende Beratung jedes Kunden. „Ich bezahle keine 1.000 Euro für einen Anzug, wenn ich vorher eine Dreiviertelstunde auf der Couch gesessen habe, um zu warten bis ich überhaupt drankomme, und dann stehen neben mir zehn andere Leute. Das ist ja auch was Emotionales! Wenn man sich einen Maßanzug machen lässt, ist das etwas anderes, als wenn dir in der Umkleidekabine der Anzug gereicht wird, du ziehst den an, der Verkäufer sagt ja, steht dir gut, und dann gehst du zur Kasse. Das ist hier viel persönlicher. Der Schneider für den Mann ist wie der Frisör für die Frau“, beschreibt Ullrich-Cattien die Situation.

Obwohl die Düsseldorfer „Modestraßen“ mit lang etablierten Unternehmen wie DOLZER, KUHN, SUIT SUPPLY und großen Ketten wie breuninger in unmittelbarer Nähe liegen, kann Ullrich-Cattien über eine mangelnde Nachfrage nicht klagen, denn ihr Konzept findet Anklang. Nach dem ersten Jahr sprach sich das Angebot von The Bloke schnell herum, seitdem wächst die Anzahl der Kunden 
pro Jahr durchschnittlich um mindestens 30 Prozent. Die zunehmende Bekanntheit entsteht also durch Mund-zu-Mund-Propaganda, aber auch durch eine durchdachte PR- und Marketingstrategie. Der Break-Even-Point war schnell erreicht, bereits im ersten Jahr nach der Gründung schrieb The Bloke operativ schwarze Zahlen.

Nach knapp fünf erfolgreichen Jahren am Standort Düsseldorf wagte The Bloke die Expansion und eröffnete ein neues Atelier in Köln. Mittlerweile umfasst das Team neben der Gründerin vier festangestellte Mitarbeiter/innen. Deren beruflicher Hintergrund ist ebenfalls vielfältig und reicht vom Designstudium über Modetechnik bis zur Schuhbranche. Wichtig für ihre Auswahl war der Hang zur Mode, insbesondere der „Fit“ zum Unternehmen. Die Mitarbeiter/innen erhalten eine intensive Schulung über mindestens ein halbes Jahr. Ihre Zufriedenheit ist dabei ein Thema, welches Ullrich-Cattien besonders wichtig ist. Zum einen arbeiten gut gelaunte und wertgeschätzte Mitarbeiter/innen besser, zum anderen sind Service und Freundlichkeit wichtige Grundbausteine des Unternehmens. Alle Mitarbeiter/innen müssen diesen Servicegedanken mittragen.

Die Motivation für Ullrich-Cattien, aus einem gut bezahlten Marketingjob in der Industrie in die Selbstständigkeit zu wechseln, war der Wunsch, autonom entscheiden und tun zu können, was man will, wie man es will und wann man es umsetzen möchte. Obwohl sie sich als Sicherheitstyp beschreibt, störte sie am Angestelltendasein in einem internationalen Großkonzern mit Sitz in den USA der immer kleiner werdende Kompetenzbereich. Weltweite Entscheidungen fielen in der USA-Zentrale, dann entschied die Europazentrale für Deutschland, sodass Manager/innen hier nur noch Details entscheiden konnten. Jetzt hat Ullrich-Cattien den Freiraum, die Dinge zu tun, die sie möchte. „Und das genieße ich auch heute noch. Ich kann entscheiden, ob ich jetzt um 22 Uhr hier sitze oder um 7 Uhr morgens.“ Die Vielfalt und der Entscheidungsfreiraum begeistern sie auch noch nach fünf Jahren im eigenen Geschäft. Trotz der hohen Arbeitsbelastung beschreibt sich die Gründerin als harmonisch und ausgeglichen. Für den Ausgleich sorgen ihre Familie, viele Freizeitaktivitäten und Sport.

\section{Spagat zwischen Etablierung und Wachstum}

Kein junges Unternehmen entwickelt sich wie das andere, jedes formt im Laufe der Zeit seine eigene Kultur hinsichtlich der Dynamik, Prozesse und Regeln. Während in der Anfangsphase oft noch kreatives Chaos herrscht, nimmt der Reifegrad mit Erfolg und Alter des Unternehmens zu. Doch bei allen wachsenden Start-ups finden sich durchgehend ähnliche Phasen mit Krisen- und Bruchstellen. Sie hat 
der Ökonom Larry E. Greiner ${ }^{4}$ in einem Modell zum Wachstum von Unternehmen zusammengefasst - mit ihren verschiedenen Lebenszyklen sowie Wendepunkten in der Entwicklung. Jede der Phasen endet jeweils in einer Krise, die dann wiederum den Übergang in die nächste Phase vorbereitet. Wachstum stellt ein Unternehmen früher oder später unter Druck, die Systeme, Prozesse und Funktionsweisen der neuen Größe anzupassen. Das Greiner-Modell beschreibt, wie die jeweilige Schwelle das Wachstum typischerweise hindert und wie sie überwunden werden kann. Daraus lassen sich charakteristische Merkmale für Entscheidungssituationen im Management ableiten. Die einzelnen Phasen des Wachstums sind in Abb. 7.1 zusammengefasst. Wie viele Modelle lässt sich auch dieses selten 1:1 auf die Praxis übertragen, dennoch treffen die Attribute der zweiten Phase am besten auf die derzeitige unternehmerische Situation von The Bloke zu. Da das GreinerModell für jede der Phasen vier bis fünf Jahre veranschlagt, ist es für das seit 2013 und damit knapp fünf Jahre bestehende Unternehmen zutreffend.

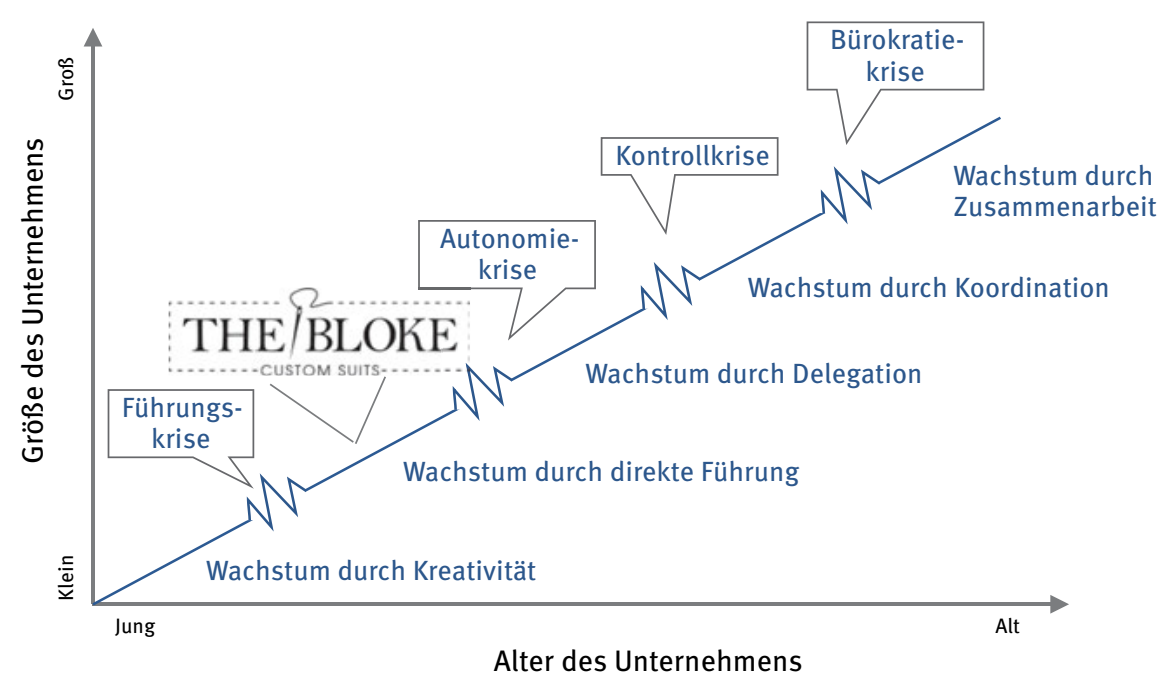

Abb. 7.1: Modell der Unternehmensentwicklung nach Greiner (Quelle: Greiner, L. E. (1997). Evolution and Revolution as Organizations Grow: A company's past has clues for management that are critical to future success. Family Business Review, 10(4), S. 397-409)

4 Vgl. Greiner, L. E. (1997). Evolution and Revolution as Organizations Grow: A company's past has clues for management that are critical to future success. Family Business Review, 10(4), S. 397-409. 
Die erste ist die Kreativitätsphase: Gründerin und Geschäftsidee standen hier im Mittelpunkt. Sie setzte alles daran, ihre Idee umzusetzen und entwickelte ein Unternehmenskonzept. The Bloke entstand mit einem minimalen Budget und in Eigenarbeit. Anfangs hatte Ullrich-Cattien den Aufbau allein geleitet und umgesetzt - von Prozessen der Designauswahl bis hin zur Kundenberatung. Sie kommunizierte das neue Unternehmen innerhalb des eigenen Netzwerkes, nahm erste regionale PR-Aktivitäten auf und bespielte ihre Social Media Kanäle. Als sie die ersten Mitarbeiter/innen einstellte, war sie keine distanzierte Managerin, sondern operativ eingebunden. Ihr Interesse galt dem Produkt und den Dienstleistungen, Steuerungs- oder Qualitätssysteme waren noch nicht etabliert. Das Wachstum erfolgte vor allem durch die Kreativität und den hohen persönlichen Einsatz der Gründerin und der ersten Mitarbeiter/innen. Die Kommunikation war informell, Gründerin und Mitarbeiter konnten Entscheidungen in spontanen Diskussionen anpassen. Im Unternehmen herrschte häufig ein kreatives Chaos. Das hochdynamische Zusammenspiel gerät am Ende der ersten Phase an seine Grenzen, so Greiner. Es kann seinem Modell zufolge zu einer Führungskrise kommen. Diese lässt sich mit dem Aufbau von Managementkompetenzen überwinden. Das hat UllrichCattien bereits erfolgreich gemeistert. Ihr betriebswirtschaftlicher Hintergrund, die gesammelte Erfahrung in der Industrie sowie ihre Nähe zu den Mitarbeitern/ innen trugen dazu in erheblichem Maße bei. Sie etablierte erste Strukturen und teilte Aufgaben ein.

Dann folgt laut Greiners Modell die Steuerungsphase: Es geht um die Ausrichtung und die funktionale Struktur des Unternehmens. Es gibt zunehmend festgelegte Abläufe und Spezialisierungen von Mitarbeitern/innen. Aufgrund der Hierarchie wird die Kommunikation formaler. Die Expansion von The Bloke in Form eines zweiten Standorts in Köln weist hier erste Ansätze in die Richtung der Steuerungsphase auf. Eine Krise entsteht, wenn das Wachstum die Motivation der Mitarbeiter/innen beispielsweise aufgrund mangelnder Autonomie in Mitleidenschaft zieht. Das ist ein wichtiges Thema für Ullrich-Cattien: ,Je größer wir werden, desto größer wird auch die Herausforderung, alles im Blick zu behalten. Also zu koordinieren und ein Gespür dafür zu behalten, dass die Mitarbeiter und Kunden zufrieden sind“ Sie leitet beide Standorte von The Bloke, was aufgrund der geografischen Nähe der beiden Städte am Rhein gut funktioniert. Bei weiteren Standorten, die entfernter vom Hauptsitz lägen, wäre es für die Gründerin schwieriger, diese ebenfalls zu leiten. Dann wäre ein verstärktes Delegieren erforderlich, das den potenziellen Übergang in die dritte Phase einleitete: die Delegationsphase. Obschon erste Strukturen erkennbar sind, macht bei The Bloke jeder noch etwas von allem. Doch Prozesse werden jetzt schon zunehmend komplexer, und wenn das Wachstum weiter wie bislang vorangeht, werden neue Strukturen erforderlich sein und Teilbereiche als Abteilung abgegeben werden müssen. 
The Bloke strebt im Rahmen des beschriebenen Phasenmodells grundsätzlich zwar Wachstum an und befindet sich in der Phase des weiteren Aufbaus. Dennoch ist es eine Herausforderung, auch die bestehenden Strukturen zu leiten und für interne Koordination sowie Sachverständnis zu sorgen. Wachstum auf Kosten einer regionalen Etablierung und internen Stabilität an bestehenden Standorten muss vermieden werden.

\section{Innovationen als Treiber für nachhaltiges Wachstum}

Dennoch kann ein Unternehmen selbst in den frühen Phasen seiner Entwicklung nicht auf Wachstum verzichten. Bei einer Expansion müssen Gründer/innen vor allem lernen, die unterschiedlichen Standorte zu verwalten sowie Flexibilität und Startkenntnisse zu bewahren, die zu ihrem ersten Erfolg geführt haben.

Einer der Schlüssel zum Erfolg eines Unternehmens, unabhängig von seiner Größe, ist Innovation. Die Entwicklung neuer Ideen ist der Motor, der Unternehmen auf dem neuesten Stand hält und sie wettbewerbsfähiger macht. Laut einer Studie von PwC glauben 93 Prozent der Führungskräfte, dass organisches Wachstum durch Innovation den größten Teil ihres Umsatzwachstums ausmacht. ${ }^{5}$

Bei kleinen Unternehmen besteht häufig das Missverständnis, Innovation sei nur größeren Unternehmen vorbehalten. Doch das ist weit verfehlt wie Richard Branson erklärt: „Kleine Unternehmen sind wendiger und mutiger und können größeren Unternehmen oft einiges über Innovationen beibringen, die ganze Branchen verändern können." ${ }^{6}$ Die Kleinen können zudem Ideen schneller umsetzen und einfacher anpassen als die Großen auf Konzernebene. Sie müssen nicht Monate oder Jahre damit verbringen, neue Ideen zu bewerten oder jedes kleine Detail über mehrere Abteilungen hinweg abzusprechen.

Mit anderen Worten: Für kleine Unternehmen bestehen weniger Hürden, wenn es um Unternehmensinnovation geht. Sie können neue Ideen schnell entwickeln und umsetzen. Daher entsteht eine Kultur, die jeden ermutigt, sich zu engagieren. So ist auch The Bloke entstanden und gewachsen. Doch seit der Gründung 2013 hat sich auf dem Düsseldorfer Markt einiges getan: Es sind viele neue Wettbewerber hinzugekommen - seien es eher traditionelle Anbieter wie die Kaufhauskette Peek \& Cloppenburg, die seit 2017 Maßkonfektion inklusive Stilberatung anbietet, oder jüngere, die auf neue Technologien wie 3-D-Scanning

5 Vgl. pwc (2018), verfügbar unter: https://www.pwc.com/im/en/assets/document/ unleashing_the_power_of_innovation.pdf.

6 Vgl. Virgin Group Holdings Limited (2014), verfügbar unter https://www.virgin.com/ entrepreneur/start-ups-join-richard-branson-for-30-year-brainstorm. 
setzen. Die bevorstehenden Aufgaben sind Etablierung und weiteres Wachstum in der beliebter werdenden Marktnische, um auch in Zukunft wettbewerbsfähig zu bleiben.

Als Lösung für ein solides Wachstum gilt es häufig, einen Ausgleich dafür zu finden, wie man auf der einen Seite bestehendes Wissen und Kompetenzen weiterhin nutzt und ausbaut, aber auf der anderen Seite auch neue Wege für eine flexible Weiterentwicklung zu gehen. Die Managementliteratur unterscheidet dabei zwischen exploitativen und explorativen Strategien, also ob vorhandene Ressourcen und Erfolgsrezepte ausgeschöpft (Exploitation) oder neue Quellen für Wachstum erschlossen werden (Exploration). Exploitation heißt: bestehendes Wissen zu nutzen und Gutes besser zu machen. Inkrementelle Neuerungen sind hier das Ziel. Das bedeutet: kleine Änderungen an bestehenden Produkten und Dienstleistungen vorzunehmen, anstatt Produkte oder Dienstleistungen vollständig zu ändern. Effizienz und Verbesserung stehen im Vordergrund, Risiken sind dabei eingeschränkt, zumindest kurzfristig. Das langfristige Risiko besteht darin, durch zu wenige, eher kleine Innovationen nicht bestehen zu können. Exploration bedeutet: neue Ideen zu generieren und radikal zu innovieren. Es geht um die Suche nach und das Experimentieren mit völlig Neuem: Ideen oder Konzepten, die sich von den derzeit auf dem Markt befindlichen Produkten, Dienstleistungen oder Betrieben deutlich unterscheiden. Diese Suche kann kostspielig und risikoreich sein. ${ }^{7}$

\section{Exploration und Exploitation als Abbild für Tradition und Moderne?}

Im Fall der Maßkonfektion kann die Abwägung beider Strategien auch zwischen der Tradition und der Moderne erfolgen. Die ganz traditionelle Variante wäre das generationsübergreifende inhabergeführte Einzelatelier, das eine relativ geringe Anzahl erlesener Kunden hat, die kaum ausbaufähig ist. Ein Problem dieses Geschäftsmodells der Maßschneiderei ist, dass die Skalierbarkeit kaum gegeben ist und Skaleneffekte hier nicht greifen. Ob nun einer oder 50 Anzüge gefertigt werden - die Stückkosten sind gleich hoch. Zudem sind Anzüge zu einem Preis ab 3.000 Euro nur für einen eher kleinen Kundenkreis interessant. Die radikale Form des Modernen entspricht dem japanische Start-up ZOZO, das seine Kunden nur noch über eine App vermisst. Ein weiteres Beispiel für die moderne Maßschnei-

7 Vgl. March, J. G. (1991). Exploration and exploitation in organizational learning. Organization Science, 2(1), S. 71-87. 
derei ist das Hamburger Unternehmen tailorjack, das eine Anleitung zum Selbstvermessen zur Verfügung stellt, sodass Kunden lediglich ihre Maße online eintragen müssen. Diese Vorgehensweise macht das Businessmodell global skalierbar, individueller Service fehlt jedoch gänzlich.

Weder das ganz traditionelle noch das völlig moderne Geschäftsmodell entspricht dem von The Bloke und passt zu den Werten der Gründerin. The Bloke vermisst zwar noch von Hand, Anzüge werden aber nicht in-house hergestellt, sondern in Marokko. Ullrich-Cattien und ihr Team können sich somit vollständig auf die Leistungen im Atelier konzentrieren: „Wir bieten den Kunden eine Menge Service-Leistungen an. Die Kunden fühlen sich aktuell gut beraten und sehr wohl bei uns. Aber ist unser Angebot das was sie auch noch in Zukunft wollen?“

Neben der Produktqualität geht es bei The Bloke also hauptsächlich um einen umfassenden Service. Daher ist es für Ullrich-Cattien derzeit undenkbar, den sich in der Branche entwickelnden radikalen Neuerungen zu folgen. Zudem sieht sie bei derartigen Modellen einen Verlust des Einkaufserlebnisses und zweifelt an der Genauigkeit der Anfertigungen.

Dass das Rad - oder hier der Anzug - nicht neu erfunden werden muss, sondern lediglich verbessert werden sollte, um nachhaltiges Wachstum in dem Nischenmarkt zu erreichen, führen die eher traditionelleren Marktspieler vor. Ein Ansatz, der auf der exploitativen Strategie beruht, ist beispielsweise der des Wettbewerbers Cove. Er bietet neben Anzügen nach Maß einen Bügelservice und Dienstleistungen rund um den perfekten Businessanzug an. Das Produktsortiment wurde um das Angebot hochwertiger Schuhe erweitert, die das Anzuggeschäft gut ergänzen. Mittlerweile bietet er zudem Maßkleider für Damen an und hat seit einiger Zeit das Geschäftsfeld um einen Online-Bereich erweitert. Dort wird alles verkauft, was ohne vorheriges Vermessen geliefert werden kann. Zuletzt hat Cove zusätzlich zwei neue Labels ausgegründet, wovon das eine Maßanzüge für junge Leute und das andere Kleidung nach Maß für Damen herstellt. Auch DOLZER und KUHN verfolgen sehr ähnliche Ansätze und konzentrieren sich primär auf die geografische Expansion. Der Online-Handel mit Accessoires mache aktuell ca. 5 Prozent des Gesamtumsatzes aus, sagt der DOLZER-Alleininhaber. Er schätzt die Tendenz zum E-Commerce als eine steigende ein. ${ }^{8}$

8 Vgl. Müller, A. (2013). Die Meister der Maße: Maßgeschneiderte Business-Mode etabliert sich und die Ansprüche der Kunden wachsen. Handelsblatt, 27.05.2013. 


\section{Exploitation als strategischer Erfolgsfaktor von The Bloke}

Generell gilt, dass die Aufrechterhaltung eines angemessenen Gleichgewichts zwischen Exploration und Exploitation ein Hauptfaktor bei der Bestimmung von Wettbewerbsfähigkeit und Wohlstand von Unternehmen ist. Das ist jedoch nicht immer der Fall und insbesondere für Nischenanbieter nichtzutreffend. Studien besagen, dass vorwiegend für Nischenanbieter im Luxussegment der Erhalt und die Nutzung von bestehendem Wissen, also Exploitation, von höherer Bedeutung ist als eine Balance zwischen explorativen und exploitativen Ansätzen. ${ }^{9}$

Die Aussage, die Nutzung bestehenden Wissens spiele eine zentrale Rolle in dem Erhalt der Wettbewerbsfähigkeit, trifft auf The Bloke zu. Ein zentraler Vorteil des jungen Unternehmens besteht darin, genau zu wissen, was die Kunden sich von einem guten Anzug erhoffen und welchem Zweck er dient. Der persönliche Kontakt zu ihnen, das „Ablesen der Wünsche von den Augen“, ermöglicht einen tieferen Einblick, als ein Scanner es jemals leisten kann, und sorgt für eine längerfristige Kundenbindung. Die Mischung von aktuellen Modetrends, Qualität und Einzigartigkeit im Stil sowie das Bestreben, der Kundennähe und der Kundenwertschätzung wieder eine übergeordnete Bedeutung zu geben, sorgen dafür, dass der Kunde nicht nur einen Anzug kauft, sondern zusätzlich das Gefühl hat, etwas Einzigartiges erhalten zu haben.

Hier spielt das bestehende Geschäftsmodell eine zentrale Rolle. Hinsichtlich der innovativen Ansätze, das Geschäftsmodell weiterzuentwickeln, ist der exploitative Ansatz tendenziell besser geeignet und entspricht eher den Wünschen der Gründerin, wie sie ihr Unternehmen führen möchte. The Bloke strebt nach Perfektion bei der Anfertigung und dem Service rund um Herrenanzüge, das heißt, diese Perfektion sollte bei der Erweiterung bestehender Service- und Produktkategorien im Vordergrund stehen. Hinterfragt man, in welchen Bereichen der exploitative Ansatz bei The Bloke greifen kann, so lässt sich die Gliederung grob in die Bereiche der Produkt- und Serviceebene einteilen sowie auf den Bereich marketingbasierter Fragestellungen erweitern.

Auf der Produktebene, also den Anzug und die Accessoires betreffend, ist sicher so bald keine radikale Veränderung im Sinne einer Produktinnovation zu erwarten. Der Kunde von The Bloke weiß, was er sich von einem Anzug wünscht, und Entsprechendes wird auch angefertigt. Für einen Maßkonfektionär ist es zudem wichtig, Neuerungen auf dem Modemarkt zu erkennen und aufzunehmen. Trends hinsichtlich Schnitte, Farbmuster und Stoffkombinationen müssen stets

9 Vgl. Cohendet, P. und Simon, L. (2015). Introduction to the special issue on creativity in innovation. Technology Innovation Management Review, 5(7). 
bekannt und in aktuellen Angeboten wiederzufinden sein. Hier werden also auch im Sinne des exploitativen Ansatzes Trends im Rahmen kleiner Änderungen am bestehenden Grundprodukt, dem Maßanzug, eingearbeitet. Ebenfalls muss eine Beratung zu diesen Neuerungen angeboten werden, das heißt, das bestehende Wissen muss ebenfalls erweitert und ausgebaut werden.

Daher betreffen exploitative Produktänderungen auch unmittelbar die Serviceebene. Hier sollte nicht nur die Beratung die bestehenden Angebote ergänzen, Erweiterungen hinsichtlich der eigentlichen Services des Maßnehmens und Anpassens könnte es ebenfalls geben, beispielsweise im Rahmen von Hausbesuchen. Dabei handelt es sich nicht um radikale Veränderung, sondern nur um eine Reaktion auf aktuelle Trends, die zusätzlich zu der Frage nach der Qualität von Anzügen auch die des „Shoppings von zu Hause aus“ aufgreifen.

Der exploitative Ansatz kann außerdem auf bestehende Marketingkonzepte angewendet werden. Wie im Rahmen der Standortwahl erläutert sollten zunächst regionale Kunden The Bloke besser kennenlernen. Dementsprechend können Social-Media-Aktionen ausgerichtet sein. Durch Kooperationen mit Bloggern, „Vloggern“ oder Instagram kann das „traditionellere Geschäftsmodell“ junge Kunden erreichen und so an regionaler Präsenz gewinnen. Explorative Ansätze sind hier weniger sinnvoll, da sich einige Social-Media-Konzepte bereits bewährt haben und sich der Trend der Nutzung und Sichtung solcher Angebote derzeit verstärkt. Ein tendenziell explorativerer Ansatz im Rahmen einer Marketingplanung wäre ein Rebranding. Er ist jedoch unter Berücksichtigung der noch frühen Phase (siehe Phasenmodell) des Unternehmens eher destruktiv, da die Marke The Bloke mit seinen Produkten und Dienstleistungen sich derzeit noch etabliert.

Anhand des bestehenden Geschäftsmodells und der strategischen Vorteile, die The Bloke aufweist, sind exploitative Innovationen für das Unternehmen von größerer Bedeutung.

\section{Heißt Erfolg immer „neu“?}

Die vorangegangenen Beschreibungen und Analysen haben gezeigt, dass viele Faktoren den Aufbau und Ausbau von Start-ups beeinflussen. Modelle wie solche, die Wachstumsphasen beschreiben, lassen sich zwar grundsätzlich auf junge Unternehmen anwenden, die Implikationen müssen jedoch immer im kontextuellen Rahmen betrachtet werden.

In der Literatur sind häufig Standpunkte zu finden, dass lediglich explorative Ansätze für wahre, nachhaltige Innovation sorgen. Doch The Bloke zeigt ein anderes Bild. Traditionelle Ideen werden hier mit einem hohen Servicegedanken 
und einer starken Kundenorientierung verfolgt, die gegen den aktuellen Trend wachsender Anonymität im E-Commerce antritt.

Welche Implikationen sind aus solchen Gegebenheiten zu ziehen? Sollte The Bloke in den Online-Handel investieren und mit dem Strom der digitalen Massen schwimmen oder weiter unter der Prämisse eines Nischenanbieters für Qualität in geringer Stückzahl stehen?

Ullrich-Cattien hat im Laufe der Jahre Erfahrungswerte hinsichtlich der Entwicklung ihres Unternehmens und der Anforderungen ihrer Kunden sammeln können. Dabei ist ihr immer wieder aufgefallen, dass insbesondere das individuelle Angebot und der persönliche Service in unserer schnelllebigen Gesellschaft eine Art „textile Pause“ und eine Belohnung für den Kunden darstellen. „Wenn ich viel Geld für meinen Anzug bezahle, dann habe ich auch das Recht darauf, dass ich eine gute, zeitintensive Beratung bekomme“, sagt die Gründerin treffend.

Der Wunsch des Kunden nach Beratung und Wertschätzung lässt sich heutzutage in wenigen Geschäften wiederfinden. Selbst Unternehmen wie HUGO BOSS, die grundsätzlich ähnlichen Tendenzen bei ihren Kunden entdecken konnten und dem Trend der Maßschneiderei folgen, sind sowohl in ihrem Ladenkonzept viel zu groß als auch stark von unternehmensinternen Vorgaben hinsichtlich der Stoffe und Schnitte beeinflusst. The Bloke bewegt sich stattdessen frei im Markt und ist weniger an Trends aus Mailand, Paris oder die Vorgaben der Geschäftsführung gebunden und kann individuell auf Kundenwünsche eingehen.

Dieser freiheitliche Gedanke zeigt sich sowohl im Tagesgeschäft von The Bloke als auch in seiner strategischen und innovativen Ausrichtung. Somit kann man dem Unternehmen nicht zwingend zu explorativen, neuen oder revolutionären Ansätzen raten, sondern muss auch hier seine Individualität betrachten und diesen zentralen Wettbewerbsvorteil über vielleicht weniger radikale Innovationsstrategien wie exploitative Ansätze erhalten.

Es gibt selbstverständlich einige Erfolgsfaktoren, die auch im Rahmen der Start-up-Forschung als Handlungsleitfaden in die Unternehmenskonzeption eingebracht werden können. Aber Ideen der Gründer/innen und ihre Passion spielen auch eine zentrale Rolle für den Erfolg. Flexibilität in der Unternehmensführung und im unternehmerischen Denken, Offenheit, Neugier und der Glaube an die eigene Idee spielen dabei für den wirtschaftlichen Erfolg teils eine wichtigere Rolle als die Analyse von Stückzahlen und die Verfolgung digitaler Trends. 


\section{(1). World}

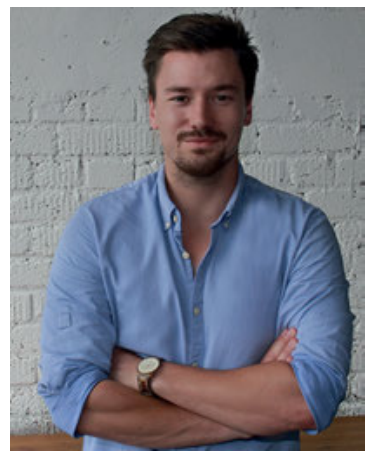

Pascal Christiaens - Gründer und Geschäftsführer der Volunteer World GmbH (Bildnachweis:

Volunteer World GmbH) 